\title{
Influence of multiple apolipoprotein A-I and $B$ genetic variations on insulin resistance and metabolic syndrome in obstructive sleep apnea
}

Xinyi $\mathrm{Li}^{1,2,3 \dagger}$, Zhihui $\mathrm{Fu}^{1,2,3+}$, Huajun $\mathrm{Xu}^{1,2,3^{*}}$, Jianyin Zou ${ }^{1,2,3}$, Huaming Zhu ${ }^{1,2,3}$, Zhiqiang Li ${ }^{4}$, Kaiming Su ${ }^{1,2,3}$, De Huai ${ }^{*}$, Hongliang $Y^{1,2,3^{*}}$, Jian Guan ${ }^{1,2,3}$ and Shankai Yin ${ }^{1,2,3}$

\begin{abstract}
Background: The relationships between apolipoprotein A-I (APOA-I), apolipoprotein B (APOB) with insulin resistance, metabolic syndrome (MetS) are unclear in OSA. We aimed to evaluate whether the multiple single nucleotide polymorphism (SNP) variants of APOA-I and APOB exert a collaborative effect on insulin resistance and MetS in OSA.

Methods: Initially, 12 APOA-I SNPs and 30 APOB SNPs in 5259 subjects were examined. After strict screening, four APOA-I SNPs and five APOB SNPs in 4007 participants were included. For each participant, the genetic risk score (GRS) was calculated based on the cumulative effect of multiple genetic variants of APOA-I and APOB. Logistic regression analyses were used to evaluate the relationships between APOA-I/APOB genetic polymorphisms, insulin resistance, and MetS in OSA.

Results: Serum APOB levels increased the risk of insulin resistance and MetS adjusting for age, gender and BMI [odds ratio $(\mathrm{OR}=3.168, P<0.001 ; \mathrm{OR}=6.098, P<0.001$, respectively]. APOA-I GRS decreased the risk of insulin resistance and MetS after adjustments ( $O R=0.917, P=0.001 ; O R=0.870, P<0.001$, respectively). APOB GRS had no association with insulin resistance $(O R=1.364, P=0.610)$, and had weak association with MetS after adjustments $(O R=1.072$, $P=0.042$ ). In addition, individuals in the top quintile of the APOA-I genetic score distribution had a lower risk of insulin resistance and MetS after adjustments ( $O R=0.761, P=0.007 ; O R=0.637, P<0.001$, respectively).
\end{abstract}

Conclusions: In patients with OSA, cumulative effects of APOA-I genetic variations decreased the risk of insulin resistance and MetS, whereas multiple APOB genetic variations had no associations with insulin resistance and weak association with MetS.

Keywords: Apolipoprotein A-I, Apolipoprotein B, Genetic risk score, Insulin resistance, Metabolic syndrome, Obstructive sleep apnea

\footnotetext{
*Correspondence: sunnydayxu2010@163.com; 18305236699@126.com; yihongl@126.com

${ }^{\top}$ Xinyi Li and Zhihui Fu contributed equally to this paper

1 Department of Otorhinolaryngology-Head and Neck Surgery, Center of Sleep Medicine, Shanghai Jiao Tong University Affiliated Sixth People's Hospital, 600 Yishan Road, Shanghai 200233, People's Republic of China ${ }^{5}$ Department of Otorhinolaryngology, Huai'an Second People's Hospital, Huai'an Hospital Affiliated to Xuzhou Medical University, 62 Huaihai South Road, Huai'an 223002, Jiangsu, People's Republic of China

Full list of author information is available at the end of the article
}

\begin{abstract}
Background
Obstructive sleep apnea (OSA), characterized by upper airway obstruction during sleep resulting in breathing pauses, intermittent hypoxia, and fragmented sleep, affects $49.7 \%$ of men and $23.4 \%$ of women and is largely undiagnosed [1]. It is also commonly recognized as an important risk factor for insulin resistance and metabolic syndrome (MetS) [2-4].
\end{abstract}

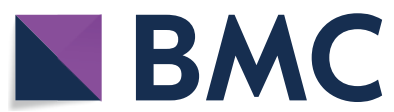

(c) The Author(s) 2020. This article is licensed under a Creative Commons Attribution 4.0 International License, which permits use, sharing, adaptation, distribution and reproduction in any medium or format, as long as you give appropriate credit to the original author(s) and the source, provide a link to the Creative Commons licence, and indicate if changes were made. The images or other third party material in this article are included in the article's Creative Commons licence, unless indicated otherwise in a credit line to the material. If material is not included in the article's Creative Commons licence and your intended use is not permitted by statutory regulation or exceeds the permitted use, you will need to obtain permission directly from the copyright holder. To view a copy of this licence, visit http://creativeco mmons.org/licenses/by/4.0/. The Creative Commons Public Domain Dedication waiver (http://creativecommons.org/publicdomain/ zero/1.0/) applies to the data made available in this article, unless otherwise stated in a credit line to the data. 
Apolipoprotein A-I (APOA-I) and apolipoprotein B (APOB) are two main lipoproteins. APOA-I is a major apolipoprotein in high-density lipoprotein cholesterol (HDL-C) and manifests antiatherogenic properties [5]. APOB is present in very low-density lipoprotein (VLDL), intermediate-density lipoprotein, and lowdensity lipoprotein cholesterol (LDL-C) and may enhance atherothrombosis $[5,6]$. Many clinical trials have revealed that APOA-I and APOB are independently associated with insulin resistance and MetS [79]. OSA is believed to be associated with APOA-I and APOB (i.e., in OSA, all sleep variables are positively correlated with the APOB/APOA-I ratio) [9]. Eight weeks of continuous positive airway pressure (CPAP) treatment can significantly decrease the APOB level [10]. Our previous study demonstrated that APOB/ APOA-I increased the risk of insulin resistance, insulin resistance play a mediator between OSA and APOB/ APOA-I [11]. However, whether APOA-I and APOB are independently associated with insulin resistance and MetS in OSA remains uncertain.

Both genetic and environmental factors play an important role in insulin resistance and MetS [12-15]. Although significant evidence links OSA to insulin resistance and MetS $[3,16]$, little is known about the roles of the genetic factors of lipoproteins involved in insulin resistance and MetS in OSA. Particularly, no current data on potential links between susceptibility genes for APOA-I and APOB and OSA-related insulin resistance and MetS are available.

Ordinarily, there is a tiny effect size of one single nucleotide polymorphism (SNP) to increase the risk of disease in a large number of variants. However, when the cumulative effect of a substantial fraction of variations reaches a certain threshold, the risk of disease is significantly increased [17]. Previous studies have used a cumulative effect model (genetic risk score, GRS) to identify risk factors of a certain disease. For example, total cholesterol (TC), total triglyceride (TG), HDL-C, and LDL-C genetic variants are associated with cardiovascular disease [18]; QT interval (measure of the time between the start of the $\mathrm{Q}$ wave and the end of the $\mathrm{T}$ wave in the heart's electrical cycle) duration genetic variants are associated with drug-induced QT prolongation [19]; and atrial fibrillation genetic variants are associated with future atrial fibrillation and stroke [20]. However, the relationships between the cumulative effects of multiple genetic variants of APOA-I, APOB, insulin resistance, and MetS in OSA remain unclear. In this study, we pooled multiple genetic variants of APOA-I and APOB to investigate the effects of APOA-I and $A P O B$ genotype on insulin resistance and MetS in the large-scale, clinical cohort study on OSA.

\section{Methods}

\section{Subjects}

Subjects who were initially suspected of having OSA were consecutively enrolled to participate in the ongoing Shanghai Sleep Heath Study (SSHS) (previously described in [21]). Subjects with non-OSA and moderate-to-severe OSA were chosen from the SSHS for an additional genomic study. Next, subjects that met the following inclusion and exclusion criteria were selected. Inclusion criteria were: older than 18 years of age without a return visit and previous treatment. Exclusion criteria were: (1) missing APOA-I and APOB data, (2) missing data on more than $15 \%$ of total SNPs, (3) regular use of lipid lowering drugs, (4) presence of a systemic disease (i.e., chronic pulmonary, renal, or hepatic failure), cancer, psychiatric disease, hyperparathyroidism, hypoparathyroidism, or polycystic ovarian syndrome; (5) other sleep disorders, such as restless leg syndrome or narcolepsy; (6) cardiovascular disease (i.e., angina, myocardial infarction, heart arrhythmia, or valvular heart disease); and (7) missing systolic blood pressure (SBP), TC, HDL-C, and fasting plasma glucose (FPG) data. Ultimately, 4007 participants were analyzed in this study that was approved by the Institutional Ethics Committee of Shanghai Jiao Tong University Affiliated Sixth People's Hospital. Written informed consent was obtained from all subjects.

\section{Anthropometric and biochemical measurements}

Waist circumference (WC) was measured at the middle of the lowest costal margin and iliac crest. Hip circumference $(\mathrm{HC})$ was measured at the widest part of the buttocks. Neck circumference $(\mathrm{NC})$ was measured at the level of the cricothyroid membrane. WC, $\mathrm{HC}$, and $\mathrm{NC}$ were measured by trained investigators following standard protocols. Body mass index (BMI) was calculated as weight (in kilograms) divided by height squared (in meters). The waist-hip ratio (WHR) was calculated as WC divided by HC (in centimeters). SBP and diastolic blood pressure (DBP) were measured in triplicate after at least a 10-min rest using an automated electronic device (Omron Model HEM-752 Fuzzy, Omron Company), and the average value of the three readings was used for analysis.

A fasting blood sample was obtained the morning after polysomnographic monitoring. FPG, TC, TG, HDLC, LDL-C, APOA-I, APOB, and apolipoprotein E were measured using an autoanalyzer (H-7600; Hitachi, Tokyo, Japan) in the hospital laboratory. Serum fasting insulin was measured using immunoassay. Homeostasis model assessment of insulin resistance (HOMA-IR) was calculated as fasting insulin $(\mu \mathrm{IU} / \mathrm{mL}) \times$ FPG $(\mathrm{mmol} / \mathrm{L}) / 22.5$. HOMA-IR $\geq 2.5$ was defined as insulin resistance [22]. Abnormal APOA-I and APOB were defined as serum 
levels $<1.20$ and $>1.10 \mathrm{~g} / \mathrm{L}$, respectively, according to diagnostic criteria of the Joint Committee for Developing Chinese Guidelines on the Prevention and Treatment of Dyslipidemia in Adults [23]. A person had metabolic syndrome if presenting three or more of the following conditions [24]: (1) TG $\geq 150 \mathrm{mg} / \mathrm{dL}$; (2) HDL-C $<40 \mathrm{mg} / \mathrm{dL}$ in men or $<50 \mathrm{mg} / \mathrm{dL}$ in women; (3) $\mathrm{SBP} \geq 130 \mathrm{mmHg}$, $\mathrm{DBP} \geq 85 \mathrm{mmHg}$, or diagnosed hypertension; (4) fasting glucose $\geq 100 \mathrm{mg} / \mathrm{dL}$ or drug treatment for type 2 diabetes; and (5) WC $\geq 90 \mathrm{~cm}$ in men or $\geq 80 \mathrm{~cm}$ in women.

\section{Polysomnographic evaluation and OSA definition}

Overnight standard polysomnography (PSG, Alice 4 or 5; Respironics Inc., Pittsburgh, PA, USA) was used to obtain objective sleep parameters. An electroencephalogram, bilateral electroculogram, chin electromyogram, electrocardiogram, nasal and oral airflow, finger pulse oximetry, chest and abdominal movements, and body posture were recorded during sleep. Apnea was defined as cessation of airflow for $\geq 10 \mathrm{~s}$, and hypopnea was defined as $\geq 50 \%$ reduction in airflow accompanied with $\geq 3 \%$ decrease in oxygen desaturation according to the 2007 American Academic Sleep Medicine criteria [25]. The severity of OSA was determined by the apnea-hypopnea index (AHI), and non-OSA, mild, moderate, and severe were defined as $\mathrm{AHI}<5,5-15,15-30$, and $\geq 30$ per hour, respectively. The oxygen desaturation index was calculated as the number of episodes of oxygen desaturation $\geq 3 \%$ per hour during sleep. The micro-arousal index was calculated as the number of arousals per hour of sleep.

\section{SNP selection, genotyping and GRS calculation}

We selected almost all of the reported APOA-I and APOB SNPs from large-scale genome-wide association studies and meta-analyses [26, 27], including 12 APOA SNPs (rs964184, rs9804646, rs12225230, rs11216162, rs5072, rs10047462, rs689243, rs10047459, rs888245, rs888246, rs625145, and rs12099358) and $30 \mathrm{APOB}$ SNPs (rs11902417, rs1042034, rs1042031, rs2678379, rs1800479, rs676210, rs693, rs1041968, rs3749054, rs673548, rs2854725, rs12713956, rs12720828, rs10199768, rs12720838, rs679899, rs570877, rs520354, rs550619, rs597331, rs531819, rs1367117, rs1800481, rs934197, rs585967, rs7575840, rs563290, rs754524, rs754523, and rs562338). After filtering those variants in our genomic database, we excluded rs531819, rs1800481, and rs585967 because the call rates were $<95 \%$. SNPs rs563290 and rs562338 were also excluded due to minor allele frequencies $<1 \%$, which failed quality control. Finally, APOA-I SNPs rs964184, rs9804646, rs10047462, and rs888246, and APOB SNPs rs1042031, rs693, rs2854725, rs1367117, and rs12713956 met linkage disequilibrium $(\mathrm{LD})<0.2$ and were analyzed in this study.

For GRS construction, we assumed an additive genetic model for each variant [28]. The weighted computation of APOA and APOB was calculated by multiplying each subject's risk allele score $(0,1$, or 2$)$ by the SNP's $\beta$ coefficient from our data; values for each locus were then summed.

\section{Statistical analysis}

Statistical analyses were performed using SPSS software (version 19.0, IBM Corp., Armonk, NY, USA). Continuous data are presented as the mean \pm standard deviation (SD) for normalized variables and as the median (interquartile range) for skewed variables. Categorical variables are shown in proportions. The Hardy-Weinberg equilibrium test was performed for each variant before association analysis using PLINK (https://zzz.bwh.harva rd.edu/plink/data.shtml). LD was performed at https:// archive.broadinstitute.org/mpg/snap/ldsearchpw.php. Differences in baseline characteristics among groups were examined using the least-significant difference test, one-way analysis of variance, the chi-squared test, the independent samples $t$-test, or the Mann-Whitney $U$ test according to the distribution characteristics of the data. Linear regressions were used to evaluate the associations between SNPs and serum APOA, APOB levels. We used logistic regression models to assess the OR of individuals in the top quintiles of the APOA and APOB GRS distributions with reference to individuals in the lowest quintile to examine the risk of moderate-to-severe OSA, insulin resistance, and MetS, both unadjusted and adjusted for age, gender, and BMI. Linear regression was used to evaluate the associations between GRS and clinical characteristics. Stepwise multivariate linear regression analysis was used to predict HOMA-IR. A two-tailed $P$ value $<0.05$ was considered statistically significant.

\section{Results \\ Baseline characteristics}

In total, 4007 eligible subjects (596 non-OSA, and 3411 moderate-to-severe OSA) were enrolled in this study (see flow chart in Fig. 1). Of the 4007 participants enrolled, 596 were non-OSA, 831 were moderate OSA and 2580 were severe OSA. Of the non-OSA subjects, the median age was 34 (range 29-43), the median HOMA-IR was 1.172 (range 1.11-2.55), median serum APOA-I was 1.092 (range 0.092-1.15) g/L, medium serum APOB was 0.77 (range $0.65-0.89$ ) $\mathrm{g} / \mathrm{L}$, the percentage of insulin resistance was $26.8 \%$, the percentage of metabolic syndrome was $27.2 \%$, the median AHI value was 2 (range 0.8-3.4) events/h. Compared with non-OSA, patients with OSA were older and had higher serum 


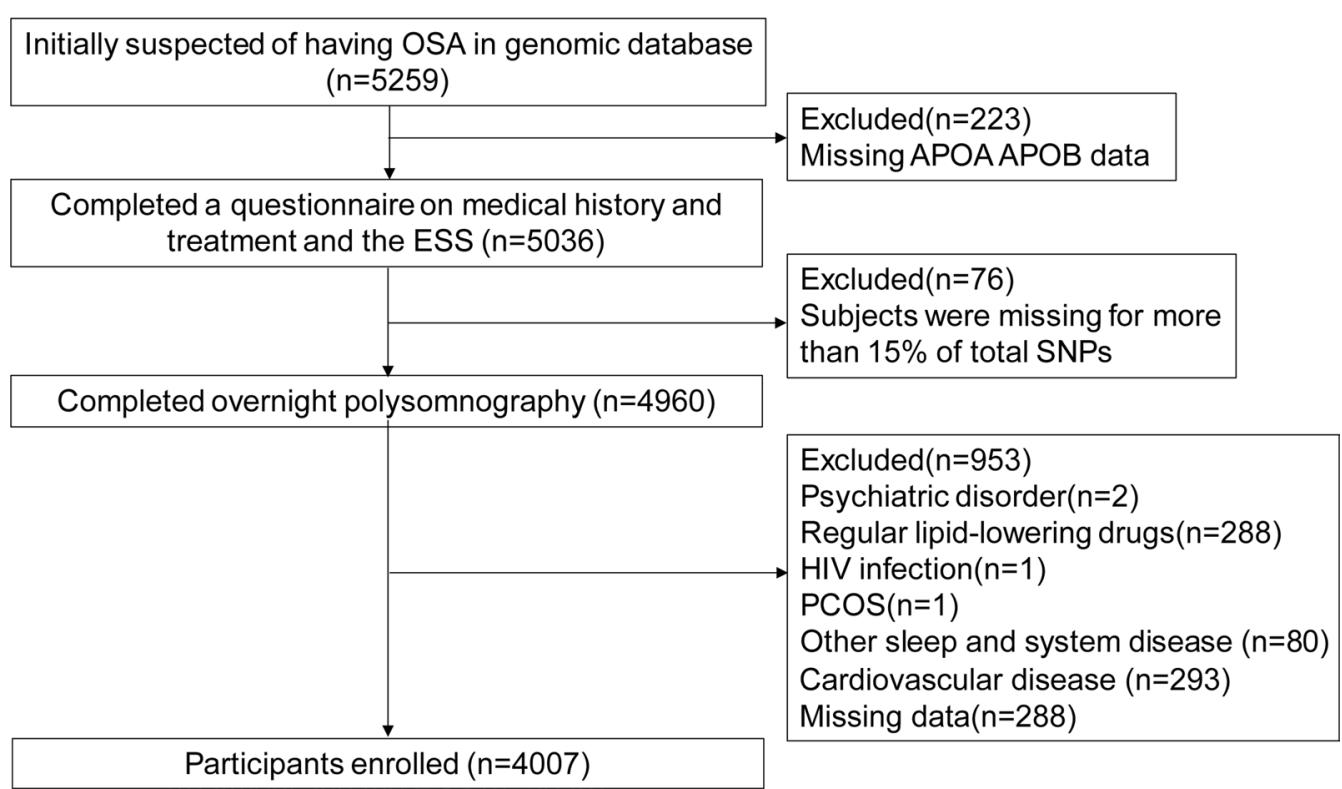

Fig. 1 Enrollment flow chart for the study population. A total of 5259 patients in the genomic database of the Shanghai Sleep Health Study cohort were included. Ultimately, 4007 patients met the inclusion criteria and were enrolled in the current study

concentrations of glucose, insulin, sleep parameters and ratio of smoking, drinking, prevalence of insulin resistance and percentage of MetS. OSA patients had higher levels of anthropometric parameter, such as SBP, DBP, BMI, NC, WC, HC, WHR. With the exception of serum APOA-I, all biochemistry parameters, demographic parameters and sleep parameters were also significantly different among the groups (Table 1). There were more subjects with insulin resistance and MetS in OSA group compared to non-OSA $(P<0.001)$. The percentages of insulin resistance in non-OSA, moderate, and severe OSA were $26.8 \%, 46.8 \%$, and $63.4 \%$, respectively. The percentages of MetS in non-OSA, moderate, and severe OSA were $27.2 \%, 50.2 \%$, and $64.3 \%$, respectively.

The basic characteristics of the SNPs and SNP's $\beta$ coefficients in GRS construction are listed in Table 2. APOA-I SNP rs964184, rs10047462, rs888246 had negative correlations ( $\beta=-0.013,-0.008,-0.014$, respectively) while rs9804646 had positive correlation with serum APOA-I levels $(B=0.015)$. APOB SNP 1042031, rs693, rs1367117 associated with APOB levels positively $(B=0.017,0.009$, 0.012, respectively), while rs2854725, rs12713956 associated with $A P O B$ levels negatively (both $B=-0.03$ ).

We also assessed the clinical characteristics of the participants in the top quintiles of the APOA GRS and APOB GRS compared with those in the bottom fifth quintiles (Additional file 1: Tables S1 and S2, respectively). The basic characteristics of insulin, insulin resistance, and TG were lower in the highest quintile of APOA
GRS than in the lowest quintile of APOA GRS, whereas HDL-C, LDL-C, APOA, and APOA/APOB were higher (all $P<0.05$; Additional file 1: Table S1). As expected, TC, $\mathrm{APOB}$, and LDL-C levels were higher in the highest quintile of APOB GRS than in the lowest quintile of APOB GRS (all $P<0.05$, Additional file 1: Table S2).

\section{Associations between common APOA-I and APOB genotypes and serum APOA-I and APOB levels}

All SNPs of APOA-I and APOB were in Hardy-Weinberg equilibrium $(P>0.05)$. The associations of SNPs with serum APOA-I and APOB levels are summarized in Table 2. APOA-I rs964184 and rs9804646 were associated with APOA-I $(\beta=-0.013, P=0.021 ; \beta=0.015$, $P=0.006)$. APOB SNP rs2854725 was significantly associated with APOB $(\beta=-0.03, P<0.001)$. APOB SNPs rs1367117 and rs12713956 were moderately associated with increased APOB levels $(\beta=0.012, P=0.008$; $\beta=-0.03, P=0.004$, respectively) (Table 2 ).

\section{Associations of serum APOA-I and APOB and their GRS with insulin resistance and MetS risks}

The associations between each SNP of APOA-I and APOB with insulin resistance and MetS are listed in Additional file 1: Table S3. APOA-I SNPs rs9804646 and rs888246 were associated with insulin resistance $(\mathrm{OR}=0.856$, 95\% confidence interval $[\mathrm{CI}]$ 0.756-0.968, $P=0.013 ; \mathrm{OR}=1.340,95 \%$ CI $1.069-1.680, P=0.011$ ) after adjustment. APOA-I SNPs rs964184, rs9804646, 
Table 1 Basic characteristics of the overall population by the severity of OSA

\begin{tabular}{|c|c|c|c|c|}
\hline Variable & Non-OSA $(n=596)$ & Moderate OSA $(n=831)$ & Severe OSA $(n=2580)$ & $P$ value \\
\hline \multicolumn{5}{|l|}{ Demographics } \\
\hline Age (years) & $34(29-43)$ & $43(35-53)$ & $41(34-51)$ & $<0.001$ \\
\hline Male (\%) & $596(100 \%)$ & 675 (81.2\%) & $2332(90.4 \%)$ & $<0.001$ \\
\hline $\mathrm{BMI}\left(\mathrm{kg} / \mathrm{m}^{2}\right)$ & $24.2(22.3-26.0)$ & $26.0(24.07-28.0)$ & $27.7(25.8-30.1)$ & $<0.001$ \\
\hline $\mathrm{NC}(\mathrm{cm})$ & $38(36-40)$ & $39(37-41)$ & $41(39-43)$ & $<0.001$ \\
\hline WC (cm) & $89(83-94)$ & $94(89-99)$ & $99(94-105)$ & $<0.001$ \\
\hline $\mathrm{HC}(\mathrm{cm})$ & $98(94-101)$ & $100(96-104)$ & 103 (99-108) & $<0.001$ \\
\hline WHR & $0.91(0.87-0.95)$ & $0.94(0.91-0.98)$ & $0.96(0.93-1.0)$ & $<0.001$ \\
\hline \multicolumn{5}{|l|}{ Biochemistry assays } \\
\hline Glucose (mmol/L) & $5.07(4.71-5.36)$ & $5.23(4.9-5.69)$ & $5.39(5.05-5.95)$ & $<0.001$ \\
\hline Insulin (uU/mL) & $7.56(5.2-11.34)$ & $10.24(7.04-14.48)$ & $12.88(8.92-18.96)$ & $<0.001$ \\
\hline HOMA-IR & $1.72(1.11-2.55)$ & $2.41(1.56-3.48)$ & $3.15(2.08-4.84)$ & $<0.001$ \\
\hline $\mathrm{SBP}(\mathrm{mmHg})$ & $120(112-128)$ & $124(115-134)$ & $127(120-138)$ & $<0.001$ \\
\hline $\mathrm{DBP}(\mathrm{mmHg})$ & $78(71-82)$ & $80(71-87)$ & $81(76-90)$ & $<0.001$ \\
\hline $\mathrm{TC}(\mathrm{mmol} / \mathrm{L})$ & $4.37(3.84-4.96)$ & $4.73(4.17-5.40)$ & $4.84(4.28-5.44)$ & $<0.001$ \\
\hline $\mathrm{TG}(\mathrm{mmol} / \mathrm{L})$ & $1.24(0.84-1.84)$ & $1.61(1.14-2.29)$ & $1.77(1.26-2.61)$ & $<0.001$ \\
\hline $\mathrm{HDL}-\mathrm{C}(\mathrm{mmol} / \mathrm{L})$ & $1.03(0.92-1.12)$ & $1.02(0.9-1.12)$ & $1(0.88-1.14)$ & $<0.001$ \\
\hline $\mathrm{LDL}-\mathrm{C}(\mathrm{mmol} / \mathrm{L})$ & $2.68(2.25-3.18)$ & $3(2.51-3.51)$ & $3.04(2.53-3.55)$ & $<0.001$ \\
\hline ApoA-I $(g / L)$ & $1.02(0.92-1.15)$ & $1.03(0.92-1.17)$ & $1.04(0.93-1.16)$ & 0.379 \\
\hline ApoB (g/L) & $0.77(0.65-0.89)$ & $0.84(0.73-0.98)$ & $0.88(0.76-1.0)$ & $<0.001$ \\
\hline ApoB/ApoA-I & $0.74(0.61-0.89)$ & $0.81(0.67-0.97)$ & $0.84(0.71-0.98)$ & $<0.001$ \\
\hline \multicolumn{5}{|l|}{ Sleep apnea } \\
\hline $\mathrm{AHI}$ & $2(0.8-3.4)$ & $21.8(18.3-25.7)$ & $58.2(45.1-70.5)$ & $<0.001$ \\
\hline Minimum $\mathrm{SaO}_{2}$ & 92 (89-95) & $83(77-87)$ & $71(62-79)$ & $<0.001$ \\
\hline ODI & $2.1(0.9-3.8)$ & $22.2(17.3-28.0)$ & $57.9(44-72.2)$ & $<0.001$ \\
\hline MAl & $14.4(10.3-22.5)$ & $22.2(14.2-31.6)$ & $36.7(21.6-56.4)$ & $<0.001$ \\
\hline \multicolumn{5}{|l|}{ Medical history } \\
\hline ESS & $7(3-10)$ & $7(4-11)$ & $11(7-15)$ & $<0.001$ \\
\hline Non-smoker, N (\%) & $372(62.4 \%)$ & $488(58.7 \%)$ & $1300(50.4 \%)$ & $<0.001$ \\
\hline Non-drinker, N (\%) & $284(47.7 \%)$ & $405(48.7 \%)$ & $1131(43.8 \%)$ & 0.024 \\
\hline IR (\%) & $160(26.8 \%)$ & $389(46.8 \%)$ & $1636(63.4 \%)$ & $<0.001$ \\
\hline Met S (\%) & 162 (27.2\%) & $417(50.2 \%)$ & $1660(64.3 \%)$ & $<0.001$ \\
\hline
\end{tabular}

and rs888246 were significantly associated with MetS $(\mathrm{OR}=1.353,95 \%$ CI 1.201-1.523, $P<0.01 ; \mathrm{OR}=0.777$, 95\% CI $0.69-0.874, P<0.01 ;$ OR $=1.274$, 95\% CI 1.024$1.586, P=0.03$, respectively) after adjustment. For APOB, only rs2854725 was associated with MetS $(\mathrm{OR}=0.829$, 95\% CI $0.718-0.956, P=0.01)$ after adjusting for age, gender, and BMI.

Serum APOA-I levels decreased the risk of insulin resistance and MetS (Table 3$) \quad(\mathrm{OR}=0.573 . P<0.001$; $\mathrm{OR}=0.131, P<0.001$ ), and had no relationship with insulin resistance after adjustment $(\mathrm{OR}=0.8233, P=0.308)$.
Serum APOA-I levels remained to decrease the risk of MetS after adjustment $(\mathrm{OR}=0.09, P<0.001)$. Serum APOB levels increased the risk of insulin resistance and MetS $\quad(\mathrm{OR}=0.573, \quad P<0.001 ; \quad \mathrm{OR}=0.131, \quad P<0.001)$, which remained after adjusting for age, gender, and BMI $(\mathrm{OR}=3.168, P<0.001 ; \mathrm{OR}=6.098, P<0.001)$. APOAI GRS decreased insulin resistance $(\mathrm{OR}=0.923,95 \% \mathrm{CI}$ 0.880-0.968, $P<0.001)$ and MetS $(\mathrm{OR}=0.886,95 \% \mathrm{CI}$ 0.845-0.929, $P<0.001)$ significantly, which remained after adjusting for age, gender, and BMI (all $P<0.001$ ). APOB GRS was not associated with insulin resistance 
Table 2 Information of each individual SNP

\begin{tabular}{|c|c|c|c|c|c|c|c|c|c|}
\hline SNP & Gene & Chromosome & Position & Minor allele & Major allele & Risk allele & $\begin{array}{l}\text { Minor allele } \\
\text { frequency }\end{array}$ & B & $P^{*}$ \\
\hline rs964184 & APOA-I & 11 & 116648917 & G & C & C & 0.216 & -0.013 & 0.012 \\
\hline rs9804646 & APOA-I & 11 & 116665079 & $\mathrm{~T}$ & C & $\mathrm{T}$ & 0.216 & 0.015 & 0.006 \\
\hline rs10047462 & APOA-I & 11 & 116722041 & G & $\mathrm{T}$ & $\mathrm{T}$ & 0.484 & -0.008 & 0.076 \\
\hline rs888246 & APOA-I & 11 & 116724232 & $\mathrm{~T}$ & C & C & 0.0052 & -0.014 & 0.16 \\
\hline rs1042031 & APOB & 2 & 21225753 & $\mathrm{~T}$ & C & $\mathrm{T}$ & 0.044 & 0.017 & 0.11 \\
\hline rs693 & APOB & 2 & 21232195 & A & G & G & 0.049 & 0.009 & 0.37 \\
\hline rs2854725 & APOB & 2 & 21237786 & G & $\mathrm{T}$ & $\mathrm{T}$ & 0.13 & -0.03 & $<0.001$ \\
\hline rs1367117 & APOB & 2 & 21263900 & A & G & G & 0.12 & 0.012 & 0.008 \\
\hline rs12713956 & APOB & 2 & 21241505 & G & A & G & 0.042 & -0.03 & 0.004 \\
\hline
\end{tabular}

APOA-I Apolipoprotein A-I, APOB Apolipoprotein B

$P^{*}$ value was adjusted for age, gender, $B M I$ as confounding factors

Table 3 Association of risk of APOA-I, APOB level and their GRS with risks of insulin resistance and MetS

\begin{tabular}{|c|c|c|c|c|c|c|c|c|c|}
\hline & Non-r & AA-IR vs HOM & & Non-M & vs MetS & & Non-OS & s moderate to & re OSA \\
\hline & OR & $95 \% \mathrm{Cl}$ & $P$ & OR & $95 \% \mathrm{Cl}$ & $P$ & OR & $95 \% \mathrm{Cl}$ & $P$ \\
\hline APOA-I GRS & 0.923 & $0.880-0.968$ & $<0.001$ & 0.886 & $0.845-0.929$ & $<0.001$ & 0.983 & $0.920-1.049$ & 0.600 \\
\hline APOA-I GRS* & 0.917 & $0.869-0.967$ & 0.001 & 0.870 & $0.827-0.916$ & $<0.001$ & 0.994 & $0.924-1.070$ & 0.874 \\
\hline APOB GRS & 1.011 & $0.950-1.076$ & 0.734 & 1.060 & $0.997-1.128$ & 0.063 & 1.011 & $0.927-1.101$ & 0.811 \\
\hline APOB GRS* & 1.364 & $1.330-1.339$ & 0.610 & 1.072 & $1.003-1.147$ & 0.042 & 1.000 & $0.907-1.104$ & 0.993 \\
\hline APOB/APOA-I & 4.551 & $3.379-6.129$ & $<0.001$ & 19.021 & $13.629-26.545$ & $<0.001$ & 7.610 & $4.886-11.852$ & $<0.001$ \\
\hline APOB/APOA-I * & 2.285 & $1.657-3.150$ & $<0.001$ & 14.488 & $10.093-20.797$ & $<0.001$ & 3.237 & $1.993-5.258$ & $<0.001$ \\
\hline APOA-I & 0.573 & $0.414-0.792$ & $<0.001$ & 0.131 & $0.093-0.184$ & $<0.001$ & 1.307 & $0.828-2.063$ & 0.250 \\
\hline APOA-I * & 0.823 & $0.566-1.196$ & 0.308 & 0.09 & $0.061-0.132$ & $<0.001$ & 1.336 & $0.788-2.267$ & 0.282 \\
\hline APOB & 6.677 & $4.654-9.578$ & $<0.001$ & 12.095 & $8.349-17.522$ & $<0.001$ & 31.683 & $18.231-55.059$ & $<0.001$ \\
\hline $\mathrm{APOB}^{*}$ & 3.168 & $2.139-4.691$ & $<0.001$ & 6.098 & $4.109-9.051$ & $<0.001$ & 8.582 & $4.653-45.830$ & $<0.001$ \\
\hline
\end{tabular}

HOMA-IR homeostasis model assessment of insulin resistance, GRS genetic risk score, Met S metabolic syndrome, APOA-I Apolipoprotein A-I, APOB Apolipoprotein B

* Adjust for age, gender, BMI

and MetS $(P>0.05)$. Subjects with OSA had higher APOB/APOA-I and APOB levels $(\mathrm{OR}=7.610,95 \% \mathrm{CI}$ 4.886-11.852, $P<0.001$; OR $=31.683$, 95\% CI 18.23155.059, $P<0.001$, respectively), even after adjusting for age, gender, and $\mathrm{BMI}$.

We also stratified APOA-I and APOB GRS into quintiles. When compared with the bottom quintile, subjects in the top quintile of the APOA-I GRS group had a lower risk of insulin resistance and MetS (Table 4) $[\mathrm{OR}=0.753$ (0.63-0.90), $P=0.002$; OR $=0.651$ (0.546$0.777), P<0.001]$, even after adjusting for age, gender, and BMI $[\mathrm{OR}=0.761(0.623-0.929), P=0.007$; $\mathrm{OR}=0.637(0.526-0.773), P<0.001]$. APOB GRS was not associated with insulin resistance $(\mathrm{OR}=1.364$, $P=0.610)$, and had weak associations with MetS $(\mathrm{OR}=1.072, \quad P=0.042)$. Linear regression analysis revealed that APOA-I GRS was associated with decreased insulin, TG, HOMA-IR, APOB/APOA-I, and elevated HDL-C, LDL-C, and APOA-I levels (all $P<0.05$, Additional file 1: Table S4), even after adjustment. APOB GRS was associated with elevated TC, LDL-C, APOB, and APOB/APOA-I levels (all $P<0.001$, Additional file 1: Table S5).

\section{Percentages of independent contributors of HOMA-IR}

To reveal the percentages of independent contributors of HOMA-IR, stepwise multivariate linear regression analysis was performed. APOA-I GRS, age, gender, and BMI were included in model 1. APOA GRS, gender, and BMI explained $0.099 \%, 0.14 \%$, and $18 \%$ of HOMA-IR, respectively (Additional file 1: Table S6). As AHI was identified as an important marker of OSA, we included AHI in model 2, APOA-I GRS, gender, BMI, and AHI explained $0.1 \%, 0.14 \%, 18 \%$, and $0.94 \%$ of HOMA-IR, respectively (all $P<0.05$, Additional file 1: Table S6). 
Table 4 Risk of OSA, insulin resistance and MetS according to quintile of APOA-I GRS and APOB GRS

\begin{tabular}{|c|c|c|c|c|c|c|}
\hline & \multicolumn{3}{|c|}{ Top vs bottom quintile unadjusted analysis } & \multicolumn{3}{|c|}{ Top vs bottom quintile adjusted analysis* } \\
\hline & OR & $95 \% \mathrm{Cl}$ & $P$ & $\mathrm{OR}^{*}$ & $95 \% \mathrm{Cl}^{*}$ & $P^{*}$ \\
\hline \multicolumn{7}{|l|}{ APOA-I GRS } \\
\hline Non-OSA vs moderate to severe OSA & 0.812 & $0.635-1.039$ & 0.098 & 0.855 & $0.654-1.118$ & 0.252 \\
\hline Non-insulin resistance vs insulin resistance & 0.753 & $0.630-0.899$ & 0.002 & 0.761 & $0.623-0.929$ & 0.007 \\
\hline Non-MetS vs MetS & 0.651 & $0.546-0.777$ & $<0.001$ & 0.637 & $0.526-0.773$ & $<0.001$ \\
\hline \multicolumn{7}{|l|}{$A P O B G R S$} \\
\hline Non-OSA vs moderate to severe OSA & 0.997 & $0.768-1.295$ & 0.984 & 0.98 & $0.738-1.301$ & 0.889 \\
\hline Non-insulin resistance vs insulin resistance & 1.019 & $0.848-1.225$ & 0.839 & 1.032 & $0.841-1.267$ & 0.761 \\
\hline Non-MetS vs MetS & 1.154 & $0.962-1.384$ & 0.123 & 1.182 & $0.971-1.439$ & 0.096 \\
\hline
\end{tabular}

OSA obstructive sleep apnea, HOMA-IR homeostasis model assessment of insulin resistance, GRS genetic risk score, MetS metabolic syndrome, $A H I$ apnoea-hypopnea index, APOA-I Apolipoprotein A-I, APOB Apolipoprotein B

* Adjust for age, gender, BMI

\section{Discussion}

Our study was the first to comprehensively examine the roles of APOA-I and APOB levels and their genetic variations on insulin resistance, MetS, and OSA using current large-scale sampling and strict data acquisition. OSA Patients had higher TC, TG, LDL-C APOB levels and APOB/APOA-I ratios than those without OSA. Subjects with OSA were more obese and had higher levels of glucose, SBP, DBP, and insulin resistance than those without OSA. Not only did serum APOA-I and APOB levels correlate with insulin resistance and MetS, but cumulative genetic variants of APOA-I and APOB also exhibited effects on insulin resistance and MetS in OSA. Individuals in the top quintile of APOA-I genetic score distributions tended to have a lower risk of insulin resistance and MetS.

Obesity is one of the most important risk factor of OSA, and the major contributing factor to the development of insulin resistance and MetS. Thus it is most likely that OSA and insulin resistance are parallel outcomes of obesity while they might not have a direct causality relationship with each other. For the past years, various experiments have been able to better unravel complex mechanisms via in-vitro and animal models, prospective observational and treatment studies revealed that the association between metabolic disease (including insulin resistance, obesity, metabolic syndrome) and OSA is bi-directional and feedforward $[4,29]$. The interrelationships among OSA, insulin resistance, MetS, dyslipidemia, obesity was multifaceted and complicated. The manuscript aimed to evaluate the influence of lipids multiple genetic variants (APOA and APOB) on insulin resistance and MetS in OSA patients using obesity as a confounding factor.

It has reported that lower APOA-I level was associated with insulin resistance in patients with impaired glucose tolerance [7] and a higher prevalence of MetS [30]. The APOB level predicted the incidence of MetS in a 5-year follow-up study [8]. The relationship between APOAI, APOB level and metabolic disease in OSA had been rarely studied. Our study suggests serum APOA-I, APOB level associated insulin resistance and MetS in OSA, and APOA-I and APOB were involved in metabolism and probably further increase cardiovascular disease risk.

APOA-I and APOB levels are not only influenced by environmental factors, such as diet and exercise, but are also subject to genetic regulation [31, 32]. Thus, APOAI and APOB genetic variations may have a causal effect on insulin resistance and MetS. Previous studies have focused on the relationship between APOA-I and APOB genetic variations and serum lipid traits [31, 33, 34]. It had been reported that there was an ethnic and gender specific association between the APOA rs964184 with lower HDL-C, higher TG and lowers APOA levels in Chinese populations [31]. The sample was small, and the results were lack of the consideration of the impact of other SNP and diseases on serum lipids levels. APOA rs964184 was also associated with serum TG levels [35, 36], metabolic syndrome [35], coronary heart disease [37], and hemorrhagic stroke risk [38]. The APOA rs964184 study on OSA and insulin resistance has not been reported yet. Our results revealed that rs964184 had negative associations with serum APOA levels but had no associations with insulin resistance in a large cross-sectional study. APOB rs1042031(EcoRI) has been widely used to study coronary heart disease (CHD) [39] and Steroid-Induced Osteonecrosis of the femoral head (SONFH) [40]. They found APOB rs1042031 confers a moderate risk for CHD [39] and increase the SONFH risk with moderate levels of evidence. APOB rs693 [41], rs2854725 [42] and rs1367117 [43, 44] were associated with serum APOB levels, further associated with familial 
hypercholesterolemia [45] and heart-related traits [46], predicted the risk of CHD [42], maternally-derived effect on BMI [43].There were fewer studies about APOA SNP rs9804646, rs10047462, rs888246 and APOB rs12713956. Data on APOA-I and APOB genetic polymorphisms in insulin resistance and MetS are still lacking. Until now, the effects of multiple genetic variants about APOA and APOB have not been studied yet, especially in a specific disease population. Our data indicate that genetic variants of APOA-I and APOB SNPs play different roles in metabolic disorders, such as APOA-I rs9804646 decreased the risk of insulin resistance and MetS, but APOA-I rs888246 increased the risk of insulin resistance and MetS. There is a tiny effect of one SNP for the disease development. Thus, we use the GRS model to study the effect of multiple genetic variations.

The GRS is a convenient way to summarize a number of genetic variants associated with an individual's genotype. The GRS does not change over time and holds the advantage that it can be used to assess the risk of metabolic dysfunction at any age from birth on. The GRS is always used in Mendelian randomization analysis to estimate the causal effect of a risk factor on an outcome [47]. This facilitates the use of genetic information, either alone or in combination, with other factors in clinical and research settings. Prospective studies have used the GRS to assess the cumulative effects of TC, TG, HDL-C and LDL-C related genetic variations on blood lipid levels, coronary events, and cardiovascular disease [18]. With the increasing availability of multiple genetic variants associated with lipids, it is becoming increasingly common to study associations with allele scores.

Our study was the first to screen 42 genetic variants and ultimately combine four APOA-I SNPs and five APOB SNPs (using the GRS model) to comprehensively examine the genetic roles of APOA-I and APOB in insulin resistance and MetS in OSA. In our study cumulative APOA-I genetic variations (APOA-I GRS) decreased the risk of insulin resistance and MetS, whereas cumulative APOB genetic variations (APOB GRS) increased the risk of MetS in OSA. The APOA-I gene is believed to be stimulated by insulin through SP-1 binding elements [48], and genetic variations of APOA-I may affect the binding site. Gene-diet interactions may also contribute to MetS [49, 50]. Because APOB SNPs are related to lipids [51, 52], the GRS of APOB may be beyond the interval of the association with insulin resistance. Future clinical trials as well as rodent studies should be designed to explore the potential mechanisms involved.

Both genetic and environmental factors are important contributors to insulin resistance. Our data indicate that environmental factors, such as BMI and AHI contribute more to HOMA-IR than do genetic variations. Genetic and environmental correlations for the same disease are complex [53]. Risk factors of OSA include obesity, age, male sex, and genetic background [54], and obesity is considered the most important risk factor $[54,55]$. Therefore, in OSA-related insulin resistance, there should be more emphasis on environmental interventions than on genetic breakthroughs.

Our study aimed to obtain high quality results by using a large sample size, laboratory-based PSG, unified serological examination, and standard questionnaires. In addition, we used multiple SNPs in a GRS model to evaluate the prediction of individual risk for metabolic disorders. However, several limitations of the present study should be noted. First, although we aimed to collect a sufficient amount of SNPs, several APOA-I and APOB SNPs may have been omitted. Furthermore, more complex genetic variants, including indels and structural variants, were not considered. The effects of SNP-SNP and gene-environment interactions were not modeled. Second, although we made efforts to minimize limitations by building our large sample population using subjects with relatively homogeneous lifestyles and ethnicity and adjusted for common confounding factors, such as age, sex, and BMI, but other more sophisticated environmental factors, such as economic status, exercise, and lifestyle, were not considered in this study. Third, we quantified the effects of multiple genetic variations, further studies on the mechanisms are still needed. The study was cross-sectional rather than prospective and community-based design, and could not provide the causative evidence. Future clinical trials, as well as animal studies of genetics-derived OSA, will better shed light on these complex mechanisms.

\section{Conclusions}

In conclusion, both the protective effects of multiple APOA-I genetic variants and damaging effects of APOB genetic variations impact the biomarkers of OSA patients.Obviously, the different cumulative effects of genes increase the complexity of metabolic disorders in OSA.

\section{Supplementary information}

Supplementary information accompanies this paper at https://doi. org/10.1186/s12986-020-00501-8.

Additional file 1: Table S1. Basic characteristics of the top vs bottom quintile APOA GRS; Table S2. Basic characteristics of the top vs bottom quintile APOB GRS; Table S3. The associations between SNPs with insulin resistance and MetS. Table S4. Linear regression of APOA GRS with clinical characteristics; Table S5. Linear regression of APOB GRS with clinical characteristics; Table S6. The stepwise multivariate linear regression model for predicting HOMA-IR. 


\begin{abstract}
Abbreviations
APOA-I: Apolipoprotein A-I; APOB: Apolipoprotein B; MetS: Metabolic syndrome; OSA: Obstructive sleep apnea; SNP: Single nucleotide polymorphism; GRS: Genetic risk score; LD: Linkage disequilibrium; HOMA-IR: Homeostasis model assessment of insulin resistance; BMI: Body mass index; TC: Total cholesterol; TG: Triglycerides; HDL-C: High-density lipoprotein cholesterol; LDL-C: Low-density lipoprotein cholesterol.
\end{abstract}

\section{Acknowledgements}

We thank all the study subjects for their participation and contribution. We acknowledge the skillful work of the entire medical staff at the Department of Otolaryngology Head and Neck Surgery \& Center of Sleep Medicine, Shanghai Jiao Tong University Affiliated Sixth People's Hospital.

\section{Authors' contributions}

Prof. HY, DH and HX had full access to all of the data in the study and took responsibility for the integrity and accuracy of the data analysis. Study design: $\mathrm{HX}, \mathrm{HY}, \mathrm{JG}$ and DH; Data collection: HX, XL, ZF, JG, JZ, HY; ZL; Statistical analysis: $H X, X L$ and ZF; Manuscript draft: $H X, X L$ and ZF. Manuscript revised: HX, JG, KS, SY. All authors read and approved the final manuscript.

\section{Funding}

National Key R\&D Program of China (2017YFC0112500); National Natural Science Foundation of China (81770987, 81700896, 81701306, 81770988); Shanghai Pujiang Program (18PJD036); Innovation Program of Shanghai Municipal Education Commission (2017-01-07-00-02-E00047); multi-center clinical research project from School of Medicine, Shanghai Jiao Tong University (DLY201502) and Shanghai Shen-Kang Hospital Management Center Project (SHDC12015101).

\section{Availability of data and materials}

All data generated or analyzed during this study can provide if needed.

\section{Ethics approval and consent to participate}

The ethics committee of Shanghai Jiao Tong University Affiliated Sixth People's Hospital approved this study according to Helsinki Declaration II. All the participants have given the informed consent before taking part in the study.

\section{Consent for publication}

Not applicable.

\section{Competing interests}

The authors declare that they have no competing interests.

\section{Author details \\ ${ }^{1}$ Department of Otorhinolaryngology-Head and Neck Surgery, Center of Sleep Medicine, Shanghai Jiao Tong University Affiliated Sixth People's Hospital, 600 Yishan Road, Shanghai 200233, People's Republic of China. ${ }^{2}$ Otolaryngological Institute of Shanghai Jiao Tong University, Yishan Road 600, Shanghai 200233, People's Republic of China. ${ }^{3}$ Shanghai Key Laboratory of Sleep Disordered Breathing, Shanghai, People's Republic of China. ${ }^{4}$ Key Laboratory for the Genetics of Developmental and Neuropsychiatric Disor- ders, Bio-X Institutes, Ministry of Education, Shanghai Jiao Tong University, Huashan Road 1954, Shanghai 200030, People's Republic of China. ${ }^{5}$ Depart- ment of Otorhinolaryngology, Huai'an Second People's Hospital, Huai'an Hospital Affiliated to Xuzhou Medical University, 62 Huaihai South Road, Huai'an 223002, Jiangsu, People's Republic of China.}

Received: 26 April 2020 Accepted: 9 September 2020 Published online: 29 September 2020

\section{References}

1. Heinzer R, Vat S, Marques-Vidal P, Marti-Soler H, Andries D, Tobback N, Mooser V, Preisig M, Malhotra A, Waeber G, et al. Prevalence of sleepdisordered breathing in the general population: the HypnoLaus study. Lancet Respir Med. 2015;3:310-8.

2. Light M, McCowen K, Malhotra A, Mesarwi OA. Sleep apnea, metabolic disease, and the cutting edge of therapy. Metabolism. 2018;84:94-8.
3. Drager LF, Togeiro SM, Polotsky VY, Lorenzi-Filho G. Obstructive sleep apnea: a cardiometabolic risk in obesity and the metabolic syndrome. J Am Coll Cardiol. 2013;62:569-76.

4. Gaines J, Vgontzas AN, Fernandez-Mendoza J, Bixler EO. Obstructive sleep apnea and the metabolic syndrome: The road to clinically-meaningful phenotyping, improved prognosis, and personalized treatment. Sleep Med Rev. 2018;42:211-9.

5. Marcovina S, Packard CJ. Measurement and meaning of apolipoprotein Al and apolipoprotein B plasma levels. J Intern Med. 2006;259:437-46.

6. Walldius $G$, Jungner I. The apoB/apoA-I ratio: a strong, new risk factor for cardiovascular disease and a target for lipid-lowering therapy - a review of the evidence. J Intern Med. 2006;259:493-519.

7. Feng X, Gao X, Yao Z, Xu Y. Low apoA-l is associated with insulin resistance in patients with impaired glucose tolerance: a cross-sectional study. Lipids Health Dis. 2017;16:69.

8. Ryoo JH, Park SK. Association of apolipoprotein B and incidence of metabolic syndrome in Korean men: a 5-years' follow-up study. Atherosclerosis. 2013:226:496-501.

9. Liu A, Cardell J, Ariel D, Lamendola C, Abbasi F, Kim SH, Holmes TH, Tomasso V, Mojaddidi H, Grove K, et al. Abnormalities of lipoprotein concentrations in obstructive sleep apnea are related to insulin resistance. Sleep. 2015;38:793-9.

10. Dorkova Z, Petrasova D, Molcanyiova A, Popovnakova M, Tkacova R. Effects of continuous positive airway pressure on cardiovascular risk profile in patients with severe obstructive sleep apnea and metabolic syndrome. Chest. 2008;134:686-92.

11. Li X, Wang F, Xu H, Qian Y, Zou J, Yang M, Zhu H, Yi H, Guan J, Yin S. Interrelationships among common predictors of cardiovascular diseases in patients of OSA: a large-scale observational study. Nutr Metab Cardiovasc Dis. 2020;30:23-322.

12. Oliveira JS, Boery R. An integrative review of associations between polymorphic variants and the metabolic syndrome. J Vasc Bras. 2018;17:141-7.

13. Manning AK, Hivert MF, Scott RA, Grimsby JL, Bouatia-Naji N, Chen H, Rybin D, Liu CT, Bielak LF, Prokopenko I, et al. A genome-wide approach accounting for body mass index identifies genetic variants influencing fasting glycemic traits and insulin resistance. Nat Genet. 2012;44:659-69.

14. Zhu Y, Zhang D, Zhou D, Li Z, Li Z, Fang L, Yang M, Shan Z, Li H, Chen J, et al. Susceptibility loci for metabolic syndrome and metabolic components identified in Han Chinese: a multi-stage genome-wide association study. J Cell Mol Med. 2017;21:1106-16.

15. Brown AE, Walker M. Genetics of insulin resistance and the metabolic syndrome. Curr Cardiol Rep. 2016;18:75.

16. Chirinos JA, Gurubhagavatula I, Teff K, Rader DJ, Wadden TA, Townsend R, Foster GD, Maislin G, Saif H, Broderick P, et al. CPAP, weight loss, or both for obstructive sleep apnea. N Engl J Med. 2014;370:2265-75.

17. Boyle EA, Li YI, Pritchard JK. An expanded view of complex traits: from polygenic to omnigenic. Cell. 2017;169:1177-86.

18. Shah S, Casas JP, Gaunt TR, Cooper J, Drenos F, Zabaneh D, Swerdlow DI, Shah T, Sofat R, Palmen J, et al. Influence of common genetic variation on blood lipid levels, cardiovascular risk, and coronary events in two British prospective cohort studies. Eur Heart J. 2013;34:972-81.

19. Strauss DG, Vicente J, Johannesen L, Blinova K, Mason JW, Weeke P, Behr ER, Roden DM, Woosley R, Kosova G, et al. Common genetic variant risk score is associated with drug-induced QT prolongation and torsade de pointes risk: a pilot study. Circulation. 2017;135:1300-10.

20. Tada H, Shiffman D, Smith JG, Sjogren M, Lubitz SA, Ellinor PT, Louie JZ, Catanese JJ, Engstrom G, Devlin JJ, et al. Twelve-single nucleotide polymorphism genetic risk score identifies individuals at increased risk for future atrial fibrillation and stroke. Stroke. 2014;45:2856-62.

21. Guan J, Yi H, Zou J, Meng L, Tang X, Zhu H, Yu D, Zhou H, Su K, Yang M, et al. Distinct severity stages of obstructive sleep apnoea are correlated with unique dyslipidaemia: large-scale observational study. Thorax. 2016:71:347-55.

22. Matthews DR, Hosker JP, Rudenski AS, Naylor BA, Treacher DF, Turner RC. Homeostasis model assessment: insulin resistance and beta-cell function from fasting plasma glucose and insulin concentrations in man. Diabetologia. 1985;28:412-9.

23. [Chinese guidelines on prevention and treatment of dyslipidemia in adults]. Zhonghua Xin Xue Guan Bing Za Zhi 2007, 35:390-419. 
24. Liu J, Grundy SM, Wang W, Smith SC Jr, Vega GL, Wu Z, Zeng Z, Wang W, Zhao D. Ethnic-specific criteria for the metabolic syndrome: evidence from China. Diabetes Care. 2006;29:1414-6.

25. Iber C, Ancoli-Israel S, Chesson A, Quan S. The AASM manual for the scoring of sleep and associated events: rules, terminology and technical specifications. Westchester: American Academy of Sleep Medicine; 2007.

26. Teslovich TM, Musunuru K, Smith AV, Edmondson AC, Stylianou IM, Koseki M, Pirruccello JP, Ripatti S, Chasman DI, Willer CJ, et al. Biological, clinical and population relevance of 95 loci for blood lipids. Nature. 2010;466:707-13.

27. Asselbergs FW, Guo Y, van Iperen EP, Sivapalaratnam S, Tragante V, Lanktree MB, Lange LA, Almoguera B, Appelman YE, Barnard J, et al. Largescale gene-centric meta-analysis across 32 studies identifies multiple lipid loci. Am J Hum Genet. 2012;91:823-38.

28. Palmer TM, Lawlor DA, Harbord RM, Sheehan NA, Tobias JH, Timpson NJ, Davey Smith G, Sterne JA. Using multiple genetic variants as instrumental variables for modifiable risk factors. Stat Methods Med Res. 2012;21:223-42.

29. Framnes SN, Arble DM. The bidirectional relationship between obstructive sleep apnea and metabolic disease. Front Endocrinol (Lausanne). 2018;9:440.

30. Yang MH, Sung J, Gwak GY. The associations between apolipoprotein $\mathrm{B}, \mathrm{A} 1$, and the $\mathrm{B} / \mathrm{A} 1$ ratio and nonalcoholic fatty liver disease in both normal-weight and overweight Korean population. J Clin Lipidol. 2016;10:289-98

31. Qiu L, Yin RX. Association of the APOA1 rs964184 SNP and serum lipid traits in the Chinese Maonan and Han populations. Lipids Health Dis. 2018;17:105.

32. Hopewell JC, Parish S, Offer A, Link E, Clarke R, Lathrop M, Armitage J, Collins R. Impact of common genetic variation on response to simvastatin therapy among 18705 participants in the Heart Protection Study. Eur Heart J. 2013;34:982-92.

33. Bandarian F, Hedayati M, Daneshpour MS, Naseri M, Azizi F. Genetic polymorphisms in the APOA1 gene and their relationship with serum HDL cholesterol levels. Lipids. 2013:48:1207-16.

34. Ordovas JM, Corella D, Cupples LA, Demissie S, Kelleher A, Coltell O, Wilson PW, Schaefer EJ, Tucker K. Polyunsaturated fatty acids modulate the effects of the APOA1 G-A polymorphism on HDL-cholesterol concentrations in a sex-specific manner: the Framingham Study. Am J Clin Nutr. 2002:75:38-46.

35. Mirhafez SR, Avan A, Pasdar A, Khatamianfar S, Hosseinzadeh L, Ganjali S, Movahedi A, Pirhoushiaran M, Mellado VG, Rosace D, et al. Zinc finger 259 gene polymorphism rs 964184 is associated with serum triglyceride levels and metabolic syndrome. Int J Mol Cell Med. 2016;5:8-18.

36. van de Woestijne AP, van der Graaf Y, de Bakker Pl, Asselbergs FW, Spiering W, Visseren FL. Rs964184 (APOA5-A4-C3-A1) is related to elevated plasma triglyceride levels, but not to an increased risk for vascular events in patients with clinically manifest vascular disease. PLoS ONE. 2014;9:e101082

37. Xu X, Li Y, Huang Y, Ye H, Han L, Ji H, Chen X, Wu N, Jiang D, Xu L, et al. Impact of gender and age on the association of the BUD13-ZNF259 rs964184 polymorphism with coronary heart disease. Anatol J Cardiol. 2018:19:42-9.

38. Zhou S, Zhao J, Wang Z, Li K, Nie S, Gao F, Sun J, Gao X, Huang Y. Association study of BUD13-ZNF259 gene rs964184 polymorphism and hemorrhagic stroke risk. Int J Clin Exp Med. 2015;8:22503-8.

39. Chen Y, Zeng J, Tan Y, Feng M, Qin J, Lin M, Zhao X, Zhao X, Liang Y, Zhang $\mathrm{N}$, Rao S. Association between apolipoprotein B EcoRI polymorphisms and coronary heart disease : a meta-analysis. Wien Klin Wochenschr. 2016;128:890-7.

40. Chen X, Zhang L, Liang D, Li J, Liu F, Ma H. Lipid Transporter activityrelated genetic polymorphisms are associated with steroid-induced osteonecrosis of the femoral head: an updated meta-analysis based on the GRADE guidelines. Front Physiol. 2018;9:1684.

41. Niu C, Luo Z, Yu L, Yang Y, Chen Y, Luo X, Lai F, Song Y. Associations of the APOB rs693 and rs 17240441 polymorphisms with plasma APOB and lipid levels: a meta-analysis. Lipids Health Dis. 2017;16:166.

42. Liu C, Yang J, Han W, Zhang Q, Shang X, Li X, Lu F, Liu X. Polymorphisms in ApoB gene are associated with risk of myocardial infarction and serum ApoB levels in a Chinese population. Int J Clin Exp Med. 2015;8:16571-7.

43. Hochner H, Allard C, Granot-Hershkovitz E, Chen J, Sitlani CM, Sazdovska S, Lumley T, McKnight B, Rice K, Enquobahrie DA, et al. Parent-of-origin effects of the APOB gene on adiposity in young adults. PLoS Genet. 2015;11:e1005573.

44. Andreotti G, Menashe I, Chen J, Chang SC, Rashid A, Gao YT, Han TQ, Sakoda LC, Chanock S, Rosenberg PS, Hsing AW. Genetic determinants of serum lipid levels in Chinese subjects: a population-based study in Shanghai, China. Eur J Epidemiol. 2009;24:763-74.

45. Karami F, Salahshourifar I, Houshmand M. The study of rs693 and rs515135 in APOB in people with familial hypercholestrolemia. Cell J. 2019;21:86-91.

46. Kulminski AM, Kernogitski Y, Culminskaya I, Loika Y, Arbeev KG, Bagley O, Duan M, Arbeeva L, Ukraintseva SV, Wu D, et al. Uncoupling associations of risk alleles with endophenotypes and phenotypes: insights from the ApoB locus and heart-related traits. Aging Cell. 2017;16:61-72.

47. Burgess S, Thompson SG. Use of allele scores as instrumental variables for Mendelian randomization. Int J Epidemiol. 2013;42:1134-44.

48. Samson SL, Wong NC. Role of Sp1 in insulin regulation of gene expression. J Mol Endocrinol. 2002;29:265-79.

49. Hosseini-Esfahani F, Mirmiran P, Daneshpour MS, Mehrabi Y, Hedayati M, Soheilian-Khorzoghi M, Azizi F. Dietary patterns interact with APOA1/ APOC3 polymorphisms to alter the risk of the metabolic syndrome: the Tehran Lipid and Glucose Study. Br J Nutr. 2015;113:644-53.

50. Hosseini-Esfahani F, Mirmiran P, Daneshpour MS, Mottaghi A, Azizi F. The effect of interactions of single nucleotide polymorphisms of APOA1/ APOC3 with food group intakes on the risk of metabolic syndrome. Avicenna J Med Biotechnol. 2017;9:94-103.

51. Chen Y, Lin M, Liang Y, Zhang N, Rao S. Association between apolipoprotein B xbai polymorphism and coronary heart disease in Han Chinese population: a meta-analysis. Genet Test Mol Biomark. 2016;20:304-11.

52. Talmud PJ, Drenos F, Shah S, Shah T, Palmen J, Verzilli C, Gaunt TR, Pallas J, Lovering R, Li K, et al. Gene-centric association signals for lipids and apolipoproteins identified via the HumanCVD BeadChip. Am J Hum Genet. 2009:85:628-42.

53. Wang K, Gaitsch H, Poon H, Cox NJ, Rzhetsky A. Classification of common human diseases derived from shared genetic and environmental determinants. Nat Genet. 2017:49:1319-25.

54. Jordan AS, McSharry DG, Malhotra A. Adult obstructive sleep apnoea. Lancet. 2014;383:736-47.

55. Hamilton GS, Joosten SA. Obstructive sleep apnoea and obesity. Aust Fam Physician. 2017:46:460-3.

\section{Publisher's Note}

Springer Nature remains neutral with regard to jurisdictional claims in published maps and institutional affiliations. 